6. M. S. Birman, On the spectrum of singular boundary problems, Mat. Sb. 55(2) 97 (1961), 125-174.

7. P. A. Rejto, On the essential spectrum of the hydrogen energy operator, Pacific J. Math. 19 (1966), 109-140.

8. Tosio Kato, Fractional powers of dissipative operators, J. Math. Soc. Japan 13 (1961), 246-274.

9. - Quadratic forms in hilbert spaces and asymptotic perturbation series, Technical Report No. 7, University of California, 1955.

10. Erik Balslev, The essential spectrum of elliptic differential operators in $L^{p}\left(R_{n}\right)$, Trans. Amer. Math. Soc. 116 (1965), 193-217.

11. Nachman Aronszajn and K. T. Smith, Theory of Bessel potentials. I, Technical Report No. 22, University of Kansas, 1959.

12. A. P. Calderón, Lebesgue spaces of differentiable functions and distributions, Proceeding of Symposia in Pure Mathematics Vol. 4, American Mathematical Society, Providence, R.I., 1961, 33-49.

13. Lars Hörmander, Linear partial differential operators, Springer, Berlin, 1963.

14. P. D. Lax and A. N. Milgram, Parabolic equations, Annals of Math. Studies, No. 33, Princeton, 1954, 167-190.

15. Erik Balslev, The essential spectrum of self-adjoint elleptic differential operators in $L^{2}\left(R_{n}\right)$, Math. Scand. (to appear).

Belfer Graduate School of Science, Yeshiva University

\title{
REPRESENTATIONS OF UNIFORMLY HYPERFINITE ALGEBRAS AND THEIR ASSOCIATED VON NEUMANNN RINGS
}

\author{
BY ROBERT T. POWERS
}

Communicated by W. Browder, February 16, 1967

Introduction. In this note we summarize the main results of a paper, Representations of uniformly hyperfinite algebras and their associated von Neumann rings, which will be published elsewhere.

A uniformly hyperfinite (UHF) algebra of class $\left\{n_{i}\right\}$ is a $C^{*}$ algebra, $\mathfrak{A}$, which contains an increasing sequence of factors, $M_{1} \subset M_{2} \subset \cdots \subset \mathfrak{A}$, of types, $\left(\mathrm{I}_{n_{1}}\right),\left(\mathrm{I}_{n_{2}}\right), \cdots$, such that $\mathfrak{A}$ is the norm closure of $\bigcup_{i=1}^{\infty} M_{i}$. It is always assumed that the integers, $n_{i} \rightarrow \infty$ as $i \rightarrow \infty$. UHF algebras have been defined and studied by Glimm [2].

If $\Pi$ is a ${ }^{*}$-representation of a UHF algebra, $\mathfrak{A}$, on a Hilbert space, then the von Neumann ring, $R=\{\Pi(\mathfrak{C})\}^{\prime \prime}$, generated by the representation algebra, $\Pi(\mathfrak{R})$, has the property that $R$ is the strong closure of an increasing sequence of type $\left(\mathrm{I}_{n}\right)$ factors. Von Neumann rings with this property will be called hyperfinite rings. It is clear that every 
hyperfinite ring can be considered as the strong closure of a representation of a UHF algebra.

Murray and von Neumann showed that all hyperfinite factors of type $\left(\mathrm{II}_{1}\right)$ are isomorphic [4]. We consider the analogous question for type (III) hyperfinite factors and arrive at the conclusion that there are uncountably many nonisomorphic type (III) hyperfinite factors.

1. Characterization of hyperfinite factors. Hyperfinite factors may be characterized by states, positive linear functionals of norm one, of UHF algebras. If $\Pi$ is a factor representation of a UHF algebra, $\mathfrak{R}$, on a Hilbert space, $\mathfrak{F}$, then any vector state, $\omega$, of $\Pi$ (i.e., any state of the form, $\omega(x)=(f, \Pi(x) f)$, for all $x \in \mathfrak{A}$, with $f \in \mathfrak{T},\|f\|=1)$ characterizes the representation, $\Pi$, up to quasi-equivalence. We recall that two representations, $\Pi_{1}$ and $\Pi_{2}$, of a $C^{*}$-algebra, $\mathfrak{A}$, are quasiequivalent if and only if there is a ${ }^{*}$-isomorphism, $\phi$, of the von Neumann ring, $\left\{\Pi_{1}(\mathfrak{H})\right\}^{\prime \prime}$, onto the von Neumann ring, $\left\{\Pi_{2}(\mathfrak{U})\right\}^{\prime \prime}$, such that $\phi\left(\Pi_{1}(x)\right)=\Pi_{2}(x)$ for all $x \in \mathfrak{A}$ (see $\left.[1, \S 5.3]\right)$. It follows that if $\Pi$ is a factor representation of a UHF algebra, $\mathfrak{A}$, and $\omega$ is a vector state of $\Pi$, then $\omega$ characterizes the hyperfinite factor, $\{\Pi(\mathfrak{X})\}^{\prime \prime}$, up to ${ }^{*}$-isomorphisms.

We consider the problem of determining the properties of those states of a UHF algebra, $\mathfrak{A}$, which induce a factor representations of $\mathfrak{A}$. It is convenient to introduce the following notation. If $S$ is a subset of a $C^{*}$-algebra, $\mathfrak{A}$, we denote by $S^{c}$ (the relative commutant of $S$ in $\mathfrak{A})$ the subalgebra of $\mathfrak{A}$ consisting of all those $x \in \mathfrak{A}$ which commute with every element of $S$ (i.e., $S^{c}=\{x \in \mathfrak{A} ; x y=y x$ for all $y \in S\}$ ). We find that factor states of a UHF algebra can be characterized by the following properties.

Theorem 1.1. Suppose $\mathfrak{A}$ is a UHF algebra and $\left\{M_{i} ; i=1,2, \cdots\right\}$ is an increasing sequence of type $\left(\mathrm{I}_{n_{i}}\right)$ factors which generate $\mathfrak{A}$. Suppose $\omega$ is a state of $\mathfrak{A}$. Then, the following conditions are equivalent.

(i) The state, $\omega$, induces a factor representation of $\mathfrak{A}$.

(ii) For every $x \in \mathfrak{A}$ there is an integer, $r>0$, depending only on $x$, such that $|\omega(x y)-\omega(x) \omega(y)| \leqq\|y\|$ for all $y \in M_{r}^{c}$.

(iii) For every $x \in \mathfrak{A}$ there is a type $\left(\mathrm{I}_{n}\right)$ factor, $M \subset \mathfrak{A}$, such that $|\omega(x y)-\omega(x) \omega(y)| \leqq\|y\|$ for all $y \in M^{c}$.

Using this result one can derive the following conditions that two factor representations of a UHF algebra be quasi-equivalent. If $\omega$ is a state of a $C^{*}$-algebra, $\mathfrak{A}$, and $M \subset \mathfrak{A}$ is a subalgebra of $\mathfrak{A}$, we denote by $\omega \mid M$ the restriction of $\omega$ to $M$.

Theorem 1.2. Suppose $\mathfrak{A}$ is a UHF algebra and $\left\{M_{i} ; i=1,2, \cdots\right\}$ 
is an increasing sequence of type $\left(\mathrm{I}_{n_{i}}\right)$ factors which generate $\mathfrak{A}$. Suppose $\Pi_{1}$ and $\Pi_{2}$ are two factor representations of $\mathfrak{A}$ and $\omega_{1}, \omega_{2}$ are vector states of the representations, $\Pi_{1}$ and $\Pi_{2}$, respectively. Then, the following statements are equivalent.

(i) $\Pi_{1}$ and $\Pi_{2}$ are quasi-equivalent.

(ii) For every $\epsilon>0$ there is an integer, $i>0$, such that $\left\|\omega_{1}\left|M_{i}^{c}-\omega_{2}\right| M_{i}^{c}\right\|$ $<\epsilon$.

(iii) For every $\epsilon>0$ there is a type $\left(\mathrm{I}_{m}\right)$ factor, $M \subset \mathfrak{A}$, such that $\left\|\omega_{1}\left|M^{c}-\omega_{2}\right| M^{c}\right\|<\epsilon$.

(iv) There is a type $\left(\mathrm{I}_{n}\right)$ factor, $N \subset \mathfrak{A}$, such that $\left\|\omega_{1}\left|N^{c}-\omega_{2}\right| N^{c}\right\|<2$.

2. Isomorphic hyperfinite rings. We will say that two representations, $\Pi_{1}$ and $\Pi_{2}$, of a UHF algebra, $\mathfrak{A}$, are algebraically equivalent if the von Neumann rings, $\left\{\Pi_{1}(\mathfrak{U})\right\}^{\prime \prime}$ and $\left\{\Pi_{2}(\mathfrak{U})\right\}^{\prime \prime}$, are ${ }^{*}$-isomorphic. Clearly, any two irreducible representations of UHF algebra are algebraically equivalent. We obtain the following necessary and sufficient condition that two ${ }^{*}$-representations of a UHF algebra be algebraically equivalent.

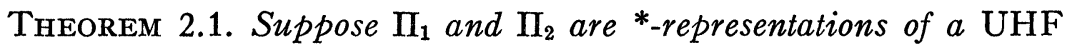
algebra, $\mathfrak{A}$, on Hilbert spaces, $\mathfrak{H}_{1}$ and $\mathfrak{H}_{2}$, respectively. Suppose at least one of these Hilbert spaces is separable. Then, the representations, $\Pi_{1}$ and $\Pi_{2}$, are algebraically equivalent if and only if there is a *automorphism, $\phi$, of $\mathfrak{A}$ such that the representations, $x \rightarrow \Pi_{1}(x)$ and $x \rightarrow \Pi(x)$ $=\Pi_{2}(\phi(x))$, are quasi-equivalent.

This result can also be stated as follows. If $\Pi_{1}$ and $\Pi_{2}$ are algebraically equivalent representations of a UHF algebra, $\mathfrak{A}$, at least one of which is on a separable Hilbert space, then there is a *-isomorphism, $\phi$, of $\left\{\Pi_{1}(\mathfrak{U})\right\}^{\prime \prime}$ onto $\left\{\Pi_{2}(\mathfrak{U})\right\}^{\prime \prime}$ such that $\phi$ maps $\Pi_{1}(\mathfrak{U})$ onto $\Pi_{2}(\mathfrak{U})$. Using this result one can show that the group of *automorphisms of a UHF algebra, $\mathfrak{A}$, acts transitively on the pure state space of $\mathfrak{A}$. Combining Theorems 1.2 and 2.1 we obtain the following corollary.

CoRollaRy 2.2. Suppose $\Pi_{1}$ and $\Pi_{2}$ are factor representations of a UHF algebra, $\mathfrak{A}$, and $\omega_{1}$ and $\omega_{2}$ are vector states of $\Pi_{1}$ and $\Pi_{2}$, respectively. Then, $\Pi_{1}$ and $\Pi_{2}$ are algebraically equivalent if and only if there is $a{ }^{*}$-automorphism, $\phi$, of $\mathfrak{A}$ such that the states, $\omega_{1}(x)$ and $\omega(x)=\omega_{2}(\phi(x))$, satisfy conditions (ii), (iii) and (iv) of Theorem 1.2.

3. Construction of nonisomorphic type (III) hyperfinite factors. Suppose $\mathfrak{A}$ is a UHF algebra of class $\left\{2^{i}\right\}$. One can show that such an algebra contains and is generated by a sequence of mutually commutative factors, $\left\{N_{r} ; r=1,2, \cdots\right\}$, of type $\left(\mathrm{I}_{2}\right)$. Each of these 
factors, $N_{r}$, is spanned by a family of matrix units, $\left\{e_{i j}^{(r)} ; i, j=1,2\right.$; $r=1,2, \cdots\}$, characterized by the following properties, $e_{11}^{(r)}+e_{22}^{(r)}=I$, $e_{i j *}^{(r)}=e_{j i}^{(r)}, e_{i j}^{(r)} e_{m n}^{(r)}=\delta_{j m} e_{i n}^{(r)}$, and $e_{i j}^{(r)} e_{n m}^{(s)}=e_{n m}^{(s)} e_{i j}^{(r)}$, for $r \neq s$, for $i, j, n, m$ $=1,2$ and $r, s=1,2, \cdots$. We define a state, $\omega_{\lambda}$, of $\mathfrak{U}$ for all $0 \leqq \lambda \leqq \frac{1}{2}$ as the unique state of $\mathfrak{A}$, such that

$$
\omega_{\lambda}\left(e_{i_{1} j_{1}}^{\left(r_{1}\right)} \cdots e_{i_{n} j_{n}}^{\left(r_{n}\right)}\right)=\lambda_{i_{1}} \delta_{i_{1} j_{1}} \cdots \lambda_{i_{n}} \delta_{i_{n} j_{n}},
$$

for all $i_{k}, j_{k}=1,2 ; r_{k} \neq r_{n}$ for $k \neq n$ and where $\lambda_{1}=\lambda$ and $\lambda_{2}=1-\lambda$. Since polynomials in the $\left\{e_{i j}^{(r)}\right\}$ are dense in $\mathfrak{A}$, it follows that the state, $\omega_{\lambda}$, is uniquely determined. From Theorem 1.1 it follows that the states, $\omega_{\lambda}$, induce factor representations, $\Pi_{\lambda}$, of $\mathfrak{A}$. The factors, $M_{\lambda}=\left\{\Pi_{\lambda}(\mathfrak{U})\right\}^{\prime \prime}$, have been studied by Glimm in [3]. In this paper Glimm shows that this family of factors, $M_{\lambda}$, is isomorphic to a family of factors constructed by Pukánszky [5] and, therefore, is able to show that for $0<\lambda<\frac{1}{2}$ the factors, $M_{\lambda}$, are of type (III). For $\lambda=0$, the state, $\omega_{\lambda}$, is pure and, therefore, induces a type $\left(I_{\infty}\right)$ factor representation of $\mathfrak{A}$. For $\lambda=\frac{1}{2}$, the state, $\omega_{\lambda}$, induces a type $\left(\mathrm{II}_{1}\right)$ factor representation of $\mathfrak{A}$. Using Corollary 2.2 we obtain the following result.

THEOREM 3.1. The representations of $\mathfrak{A}$ induced by the states, $\omega_{\lambda}$ and $\omega_{\lambda^{\prime}}$, are algebraically equivalent if and only if $\lambda=\lambda^{\prime}$, i.e., the factors, $M_{\lambda}$, are mutually nonisomorphic for distinct $\lambda, 0 \leqq \lambda \leqq \frac{1}{2}$. Therefore, there are uncountably many nonisomorphic type (III) hyperfinite factors.

\section{REFERENCES}

1. J. Dixmier, Les $C^{*}$-algèbres et leurs represéntations, Gauthier-Villars, Paris, 1964.

2. J. Glimm, On a certain class of operator algebras, Trans. Amer. Math. Soc. 95 (1960), 318-340.

3. - Type I $C^{*}$-algebras, Ann. of Math. 73 (1961), 572-612.

4. F. Murray and J. von Neumann, On rings of operators. IV, Ann. of Math. 44 (1943), 716-808.

5. L. Pukánszky, Some examples of factors, Publ. Math. Debrecen. 4 (1956), $135-156$.

Palmer Physical laboratory, Princeton University 